\title{
Star-burst regions in the LMC ${ }^{\star}$ (Research Note)
}

\author{
E. Livanou ${ }^{1}$, M. Kontizas ${ }^{1}$, I. Gonidakis ${ }^{1}$, E. Kontizas ${ }^{2}$, F. Maragoudaki ${ }^{1}$, S. Oliver ${ }^{3}$, A. Efstathiou ${ }^{4}$, and U. Klein ${ }^{5}$ \\ 1 Department of Astrophysics Astronomy \& Mechanics, Faculty of Physics, University of Athens, 15783 Athens, Greece \\ e-mail: elivanou@phys.uoa.gr \\ 2 Institute for Astronomy and Astrophysics, National Observatory of Athens, PO Box 20048, 11810 Athens, Greece \\ 3 Astronomy Centre, Department of Physics \& Astronomy, University of Sussex, Brighton, BN1 9QJ, UK \\ ${ }^{4}$ Department of Computer Science and Engineering, Cyprus College, 6 Diogenes Str, 1516 Nicosia, Cyprus \\ 5 Radioastronomisches Institut der Universitat, Bonn, Auf dem Hugel 71, 53121 Bonn, Germany
}

Received 25 February 2004 / accepted 30 November 2005

\section{ABSTRACT}

Aims. Filamentary structures of early type stars are found to be a common feature of the Magellanic Clouds formed at an age of about $0.9-2 \times 10^{8}$ yr. As we go to younger ages these large structures appear fragmented and sooner or later form young clusters and associations. In the optical domain we have detected 56 such large structures of young objects, known as stellar complexes in the LMC for which we give coordinates and dimensions. We also investigate star formation activity and evolution of these stellar complexes and define the term "starburst region".

Methods. IR properties of these regions have been investigated using IRAS data. A colour-magnitude diagram (CMD) and a two-colour diagram from IRAS data of these regions ware compared with observations of starburst galaxies and cross-matching with HII regions and SNRs was made. Radio emission maps at 8.6-GHz and the $\mathrm{CO}(1 \rightarrow 0)$ line were also cross correlated with the map of the stellar complexes. Results. It has been found that nearly $1 / 3$ of the stellar complexes are extremely active resembling the IR behaviour of starburst galaxies and HII regions. These stellar complexes illustrating such properties are called here "starburst regions". They host an increased number of HII regions and SNRs. The main starburst tracers are their IR luminosity ( $F_{60}$ well above $\left.5.4 \mathrm{Jy}\right)$ and the 8.6-GHz radio emission. Finally the evolution of all stellar complexes is discussed based on the $\mathrm{CO}$ emission.

Key words. Magellanic Clouds - galaxies: starburst - stars: formation

\section{Introduction}

Due to its proximity to our Galaxy and the SMC, the LMC offers two important advantages: i) the possibility to study its stellar content down to low stellar masses and ii) the possibility to follow the consequences of the close interaction, about $2-4 \times 10^{8}$ yr ago (Gardiner \& Noguchi 1996; Kunkel et al. 2000), with the SMC. Stellar complexes are defined as large structures (clump like) in galaxies dominated by recently formed stellar component mixed with very young clusters, stellar associations and gas (Martin et al. 1976; van den Bergh 1981; Elmegreen \& Elmegreen 1983, 1987; Feitzinger \& Braunsfurth 1984; Ivanov 1987; Larson et al. 1988; Efremov 1989, 1995; Larson et al. 1992; Efremov \& Chernin 1994; Elmegreen et al. 1994; Kontizas et al. 1994; Battinelli et al. 1996; Elmegreen \& Efremov 1996; Kontizas et al. 1996). So

* Tables 2 and 3 are only available in electronic form at http://www . edpsciences.org far they have been detected as regions with young stellar component and enhanced stellar number density, compared to the surrounding region. In the LMC stellar complexes have been detected using UK Schmidt plates (Maragoudaki et al. 1998). In the optical domain we have detected 56 large structures of young objects. These stellar complexes are associated with the already known nine Shapley constellations (Shapley 1956), which are referred to as large stellar structures of early type supergiants.

Helou (1986) found that the IRAS $\log \left(F_{60} / F_{100}\right)$ versus $\log \left(F_{12} / F_{25}\right)$ colour-colour diagram of "normal galaxies" shows a distribution that extends continuously from the cool $(\sim 20 \mathrm{~K})$ relatively constant "cirrus" emission from the neutral medium to the warmer $(\sim 40-50 \mathrm{~K})$ emission from the active, starburst end.

IRAS far-IR fluxes for $6 \mathrm{HII}$ regions in the LMC have been studied by DeGioia-Eastwood (1992). These regions are the sites of massive star formation, where the radiative heating 
source is young stars rather than the general interstellar radiation field. Such regions are expected to lie in stellar complexes and/or to be the stage just before the stellar complex is formed.

The highly structured diffuse X-ray emission of the LMC has been imaged in detail by ROSAT. The brightest regions are found east of LMC X-1 and in the 30 Doradus region. The latter is strikingly similar to the optical picture. There is a strong correlation between diffuse features in the X-ray image and ESO colour images of the LMC in visible light. Bright knots in the X-ray map correspond to HII emission in the optical (Westerlund 1997). The distribution of the SNRs in the LMC shows (i) a clumping of objects in the 30 Doradus region, (ii) several remnants within the Bar of the LMC and (iii) that the remainder of the remnants are found in super-associations. Most SNRs in the Bar are in regions where many young clusters are located, clusters as young as $\leq 10 \mathrm{Myr}$ (Westerlund 1997).

The LMC has been well studied in the radio continuum, which is connected to the formation of new and massive stars. Fukui et al. $(1999,2001)$ found that the molecular clouds in the LMC have a good correlation with the youngest ( $\$ 10 \mathrm{Myr}$ ) stellar clusters, while there is little spatial correlation of these clouds with SNRs or with the older stellar clusters.

In this paper we describe how the stellar complexes were detected, using optical data and we give their location and dimensions. The total flux of each structure is presented. Their derived IR properties are compared to IR properties of galaxies in order to identify the distinction between ordinary stellar complexes and active star formation regions. Possible connection of the complexes determined here with known X-ray detected SNRs and candidates as well as HII regions is discussed. Finally, their correlation between high resolution radio data at 8.6- $\mathrm{GHz}$ and $\mathrm{CO}$ emission is examined, in order to determine the activity of the complexes and their evolution.

\section{Optical data}

The large-scale structures in nearby galaxies require extended field observational material in order to be treated homogeneously. Therefore we used direct photographic plates taken with the UK $1.2 \mathrm{~m}$ Schmidt Telescope, in various wavebands: $U, R$ and HeII ( $\lambda=4686 \AA)$ down to a magnitude limit of 19-20 mag. These plates were digitised using the fast measuring machines APM and SuperCosmos. The derived data were produced as catalogues of detected stellar images. Star counts were performed on the above catalogues in order to derive isopleth contour maps of the LMC in the various wavebands and magnitude slices, to trace the differences between faint and brighter stellar populations. Star counts, in the optical wavelength range, of large areas in the LMC have revealed 56 groupings of young stars, known as stellar complexes (with dimensions from $150 \mathrm{pc}$ to $\sim 1500 \mathrm{pc}$ ). Their coordinates, dimensions and properties are given in Table 2, whereas their ages span from a few $\times 10^{8} \mathrm{yr}$ to very young $\left(\sim 10^{6} \mathrm{yr}\right)$. The ages are derived from their stellar component, as described by Maragoudaki et al. (1998), which consist of early type stars (O, B, A), dust, young clusters and associations, very often in groups. The parental density fluctuations, which were

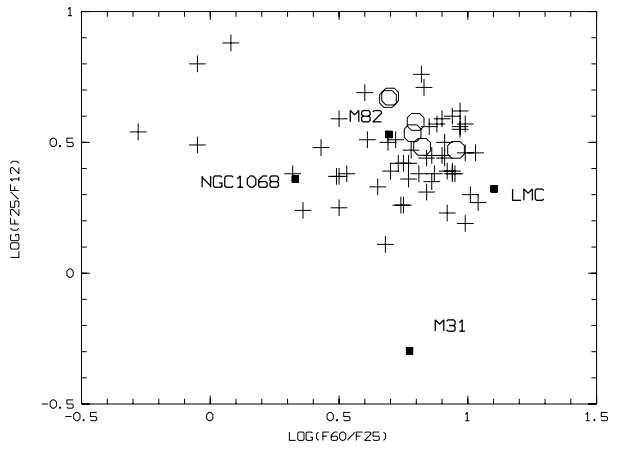

Fig. 1. IRAS colour-colour diagram for the detected stellar complexes. The black-filled squares mark the observed colours of the LMC (irregular galaxy) and three typical galaxies: M 31 (disk), M 82 (starburst) and NGC 1068 (AGN). The circles mark LMC HII regions.

fragmented into stellar complexes can be explained as a result of the close encounter of the SMC with the LMC that happened a few $\times 10^{8}$ yr ago (Gardiner \& Noguchi 1996; Kunkel et al. 2000).

\section{IR data}

IRAS all-sky survey Images taken at 12, 25, 60 and $100 \mu \mathrm{m}$ have been obtained from SkyView (http://skyview.gsfc.nasa.gov/skyview.html) for the whole area of the LMC to study the IR properties of the stellar complexes detected in the optical domain. From the IRAS catalogues we calculated a total flux for each complex as the sum of the fluxes corresponding to the pixels, which cover the predetermined complex area. These total fluxes were corrected from the background. The background value was assumed as the average of four flux/pixel values at the four rather clear corners of each frame, for all IRAS wavelengths respectively. The background corrected total fluxes were averaged to a mean value (flux/pixel in $\mathrm{MJy} / \mathrm{sr}$ ) for all complexes so that they can be more easily compared with the literature.

Figure 1 shows the IRAS two-colour diagram of $\log \left(F_{25} / F_{12}\right)$ vs. $\log \left(F_{60} / F_{25}\right)$ for the detected stellar complexes. The black-filled squares represent the integrated values for NGC 1068, M 82, M 31 and LMC respectively, indicating the loci of different types of galaxies on this diagram. NGC 1068 is a prototype AGN (Rowan-Robinson \& Crawford 1989), M 82 is a prototype starburst galaxy, while M 31 is a typical disk galaxy (Rice et al. 1988). The LMC HII regions of DeGioia-Eastwood (1992) are plotted with circles. We notice that many stellar complexes lie around the location of M 82, a prototype starburst, and the HII regions revealing enhanced star formation activity.

The IRAS flux densities in $\log F_{60}$ versus the IRAS colour $\log \left(F_{60} / F_{100}\right)$ for all the large structures detected here are illustrated in Fig. 2. Lehnert \& Heckman (1996) seeking to improve our understanding of the range of physical processes occurring within IR selected galaxies, found that the starburst galaxies are IR "warm" if $F_{60} / F_{100} \geq 0.4\left(\log \left(F_{60} / F_{100}\right) \geq\right.$ $-0.39)$ and IR "bright" if $F_{60} \geq 5.4 \mathrm{Jy}\left(\log \left(F_{60}\right) \geq 0.73\right)$. From Fig. 2 it is obvious that nearly $1 / 3$ of the detected stellar complexes fulfil the criteria defined by Lehnert \& Heckman (1996) 


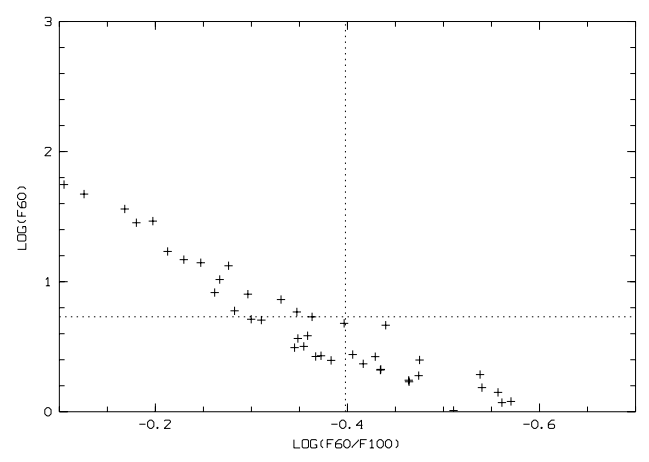

Fig. 2. IRAS flux density in $\log F_{60}$ versus IRAS colour $\log \left(F_{60} / F_{100}\right)$ for all the structures detected here. Two lines are plotted which represent $F_{60}=5.4 \mathrm{Jy}$ and $F_{60} / F_{100}=0.4$. The upper left area indicates the loci of very active star forming regions.

for the starburst galaxies. The three upper points correspond to regions in 30 Doradus (complexes C17, A21, A24), where there is the highest concentration of SNRs. The 15 structures that have $\log \left(F_{60} / F_{100}\right) \geq-0.39$ and $\log \left(F_{60}\right) \geq 0.73$, resemble the behaviour of starburst galaxies, whereas the rest 41 do not show enhanced star formation activity.

\section{Radio and CO data}

We used the recently published 8.6-GHz map of Dickel et al. (2005), with its angular resolution of $20^{\prime \prime}$ and linear resolution $\sim 5 \mathrm{pc}$ and the $\mathrm{CO}(1 \rightarrow 0)$ line that was fully mapped by Fukui et al. (2001), with angular resolution of 2.6' corresponding to a spatial resolution of $\sim 40$ pc. In Fig. 3 we have superimposed the stellar aggregates, complexes and super-complexes (yellow lines) onto the radio $8.6-\mathrm{GHz}$ (grey-scale) and $\mathrm{CO}$ (red contours) map. The time sequence evident in this figure is such that the molecular gas (CO) traces regions of ongoing and (near-) future star formation, the ionised gas $(8.6-\mathrm{GHz})$ locates ongoing and somewhat evolved star formation $\left(10^{7} \sim 10^{8} \mathrm{yr}\right)$. We examined the correlation between $8.6-\mathrm{GHz}$ radio emission, the $\mathrm{CO}$ emission and the location of the 56 stellar complexes (Table 2, Cols. 8 and 9).

We notice that the majority (13 out of 15 ) of the regions with $F_{60}>5.4 \mathrm{Jy}$, coincide well with the $8.6-\mathrm{GHz}$ radio emission. The existence of $\mathrm{CO}$ emission in seven of them, reveals future star formation activity. The rest 2 , that lack both $8.6-\mathrm{GHz}$ and $\mathrm{CO}$ radiation, are actually found to have values of $F_{60}$ very close to $5.4 \mathrm{Jy}$. Considering the 41 regions with $F_{60}<5.4 \mathrm{Jy}$, the opposite behaviour is revealed. 36 out of 41 regions do not exhibit significant amount of 8.6-GHz radiation, while the remaining 5 that do exhibit $8.6-\mathrm{GHz}$ radiation, are found to have $F_{60}$ very close to $5.4 \mathrm{Jy}$.

The CO emission is indicative of on-going and possible future star formation activity and there is no pattern revealed regarding the $F_{60}$ of the complexes. 16 out of the 56 complexes have significant $\mathrm{CO}$ emission, indicating possible future star formation activity and only 10 of them are correlated with 8.6-GHz emission as well.

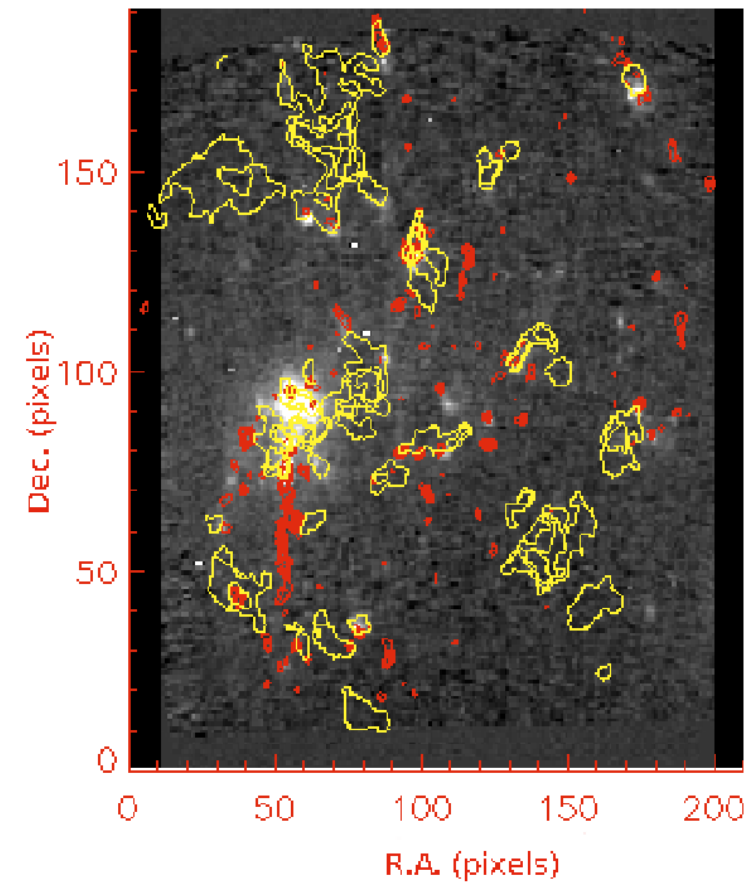

Fig. 3. Maps of the LMC at $8.6 \mathrm{GHz}$ (grey-scale) and in the $\mathrm{CO}(1 \rightarrow 0)$ line (red contours), with the stellar complexes superimposed with yellows lines.

\section{Discussion and conclusions}

From optical observations, 56 stellar complexes are revealed in an area of $6 \times 7 \mathrm{deg}^{2}$ of the LMC. The IR properties are studied using the IRAS fluxes. The ratio of the flux densities $F_{60} / F_{100}$, the flux density $F_{60}$ as discussed above, the 8.6- $\mathrm{GHz}$ and $\mathrm{CO}$ radio emission, will be used in order to classify these complexes.

As mentioned in Sect. 3, Lehnert \& Heckman (1996) showed that starburst galaxies are IR "warm" and "bright". In the same manner, we adopt the equivalent term "starburst regions" for complexes that are found to fulfil these criteria, namely $F_{60} / F_{100} \geq 0.4$ and $F_{60} \geq 5.4 \mathrm{Jy}$. In addition, complexes that do not fulfil the previously stated criteria will be called "active complexes".

A more detailed study of high resolution $8.6-\mathrm{GHz}$ map (Fig. 3) revealed that all complexes with $F_{60}$ well above the $5.4 \mathrm{Jy}$ limit are very well correlated with the 8.6-GHz radio emission. In contrast, complexes with $F_{60}$ well below the $5.4 \mathrm{Jy}$ limit lack $8.6-\mathrm{GHz}$ emission. However, 7 out of the 56 complexes (A5, A6, A7, C5, C7, C22 and A31) have $F_{60}$ close to the classification limit and deviate from the above behaviour. Complex C22, for example, is well correlated with $8.6-\mathrm{GHz}$ emission; its $F_{60}(5.07 \pm 0.20 \mathrm{Jy})$ though is below the accepted limit. In contrast $\mathrm{A} 31$ has $F_{60}=5.98 \pm 0.20 \mathrm{Jy}$, which would classify it as a starburst region; however, there is no $8.6-\mathrm{GHz}$ emission associated with it. Since the $F_{60}$ criterion is not trustworthy for these regions, we additionally use the $8.6-\mathrm{GHz}$ emission to classify the complexes as "starburst candidates" or "active complexes candidates" depending on its existence. Hence, using the 8.6-GHz radiation as an additional tracer in tandem with the $F_{60}$, we can classify the stellar complexes into 
Table 1. Complexes characterisation.

\begin{tabular}{lccc}
\hline \hline Complex type & $\begin{array}{c}F_{60} \\
(\mathrm{Jy})\end{array}$ & $\begin{array}{c}8.6 \\
(\mathrm{GHz})\end{array}$ & $\begin{array}{c}\text { No. of } \\
\text { Complexes }\end{array}$ \\
\hline starburst & $F_{60}>5.4$ & yes & 13 \\
starburst candidate & $F_{60} \lesssim 5.4$ & yes & 5 \\
active complex candidate & $F_{60} \gtrsim 5.4$ & no & 2 \\
active complex & $F_{60}<5.4$ & no & 36 \\
\hline
\end{tabular}

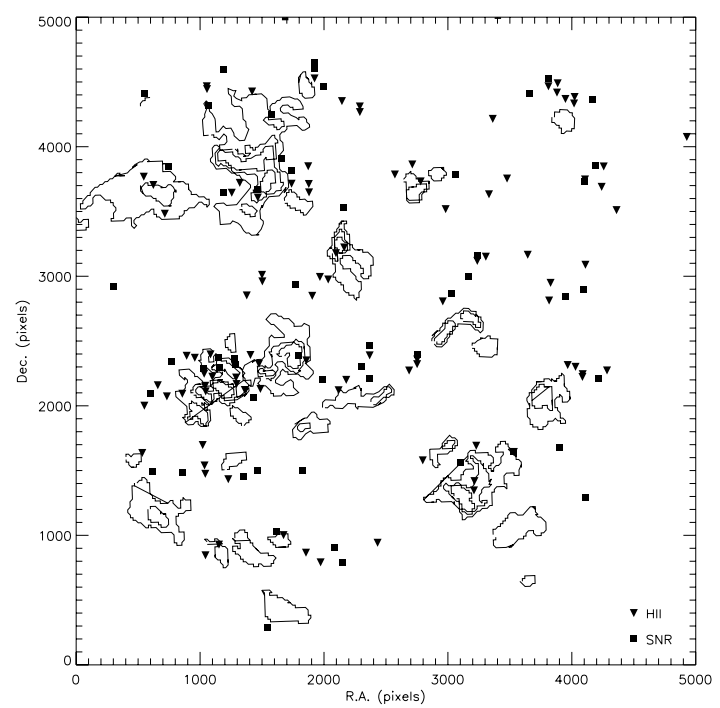

Fig. 4. The HII regions (triangles) and SNRs (squares), plotted over the stellar complexes as defined here.

13 starbursts, 5 starburst candidates, 2 active complexes candidates and 36 active complexes as seen in Table 1 . The characterisation of each complex is given in Table 2, Col. 10.

Moreover, a discrimination concerning the evolution of all the complexes was attempted based on the $\mathrm{CO}$ data. More than half of the starburst and starburst candidate regions show enhanced $\mathrm{CO}$ emission indicating ongoing and future evolution, while the rest of them are thought to be more or less evolved. Regarding the active stellar complexes (and the two candidates), we found only 5 of them with significant amount of $\mathrm{CO}$ emission, giving additional indication of current star formation and revealing potential future starbursts. The lack of $\mathrm{CO}$ in the rest of them could indicate that these complexes have never been starbursts or that their starburst activity previously exhausted the molecular gas.

In Fig. 4 we plotted the known SNRs and HII regions based on the literature (Williams et al. 1999; Haberl \& Pietsch 1999; Filipovic et al. 1998; Sasaki et al. 2000) over the stellar complexes with triangles and squares respectively. The overplotted HII regions show an increased concentration in and around the very active star forming regions, as expected. Most of the defined starburst areas (and the candidates) are associated with SNRs. The detected complexes are also cross-matched with the catalogue of stellar associations (Gouliermis et al. 2003) and that of nebulae in the LMC (Davies et al. 1976). The majority of the complexes are loci of stellar associations, whereas all of them are associated with a large number of nebulosities (Table 2).

Acknowledgements. The authors wish to thank the British Council and the General Secretariat of Research and Technology for financial support. M. Kontizas would like to thank the University of Athens (ELKE) for partial financial support. Finally M. Kontizas, E. Livanou and I. Gonidakis are very much indebted to the Ministry of Education for the financial support through the "Pythagoras II" program.

\section{References}

Battinelli, P., Efremov, Yu. N., \& Magnier, E. 1996, A\&A, 314, 51 DeGioia-Eastwood, K. 1992, ApJ, 397, 542

Davies, R. D., Elliot, K. H., \& Meaburn, J. 1976, MmRAS, 81, 89

Dickel, J. R., McIntire, V. J., Gruendl, R. A., \& Milne, D. K. 2005, ApJ, L29, 790

Efremov, Yu. N. 1989, Sov. Sci. Rev., Section E, 7, Part 2

Efremov, Yu. N. 1995, AJ, 110, 2757

Efremov, Yu. N., \& Chernin, A. D. 1994, Vistas Astron., 38, 165

Elmegreen, B. G., \& Efremov, Yu. N. 1996, ApJ, 466, 802

Elmegreen, B. G., \& Elmegreen, D. M. 1983, MNRAS, 203, 31

Elmegreen, B. G., \& Elmegreen, D. M. 1987, ApJ, 320, 182

Elmegreen, D. M., Elmegreen, B. G., Lang, C., \& Stephens, C. 1994, ApJ, 425, 57

Feitzinger, J. V., \& Braunsfurth, E. 1984, A\&A, 139, 104

Filipovic, M. D., Pietsch, W., Haynes, R. F., et al. 1998, A\&AS, 127, 119

Fukui, Y., Mizuno, N., Yamagushi, R., et al. 1999, PASJ, 51, 745

Fukui, Y., Mizuno, N., Yamaguchi, R., Mizuno, A. \& Onishi, T. 2001, PASJ, 53, 41

Gardiner, L. T., \& Noguchi, M. 1996, MNRAS, 278, 191

Gouliermis, D., Kontizas, M., Kontizas, E., \& Korakitis, R. 2003, A\&A, 405, 111

Haberl, F., \& Pietsch, W. 1999, A\&AS, 139, 277

Helou, G. 1986, ApJ, 311, L33

Ivanov, G. R. 1987, Ap\&SS, 136, 113

Kontizas, M., Kontizas, E., Dapergolas, A., Argyropoulos, S., \& Bellas-Velidis, I. 1994, A\&AS, 107, 77

Kontizas, M., Maravelias, S. E., Kontizas, E., Dapergolas, A., \& Bellas-Velidis, I. 1996, A\&A, 308, 40

Kunkel, W. E., Demers, S., \& Irwin, M. J. 2000, AJ, 119, 2789

Larson, R. B. 1988, in Galactic and extragalactic star formation, ed. R. Pudritz, \& M. Fich, 459

Larson, R. B. 1992, in Star formation in stellar systems, ed. G. Tenorio-Tagle et al. (Cambridge: Cambridge Univ. Press)

Lehnert, M. D., \& Heckman, T. M. 1996, ApJ, 472, 546

Maragoudaki, F., Kontizas, M., Kontizas, E., Dapergolas, A., \& Morgan, D. H. 1998, A\&A, 338, L29 (Paper I)

Martin, N., Prevot, L., Rebeirot, E., \& Rousseau, J. 1976, A\&A, 51, 31

Rice, W., Lonsdale, C. J., Soifer, B. T., et al. 1988, ApJS, 68, 91

Rowan-Robinson, M., \& Crawford, J. 1989, MNRAS, 238, 523

Sasaki, M., Haberl, F., \& Pietsch, W. 2000, A\&AS, 143, 391

Shapley, H. 1956, Amer. Scientist, 44, 73

van den Bergh, S. 1981, A\&AS, 46, 79

Williams, R. M., Chu, Y., Dickel, J. R., et al. 1999, ApJS, 123, 467

Westerlund, B. E. 1997, The Magellanic Clouds, Camb. Astr. Ser., 29 


\section{Online Material}


Table 2. List of the LMC stellar complexes. Column 1 indicates the adopted identification name. A denotes aggregate, C complex and SC supercomplex. For a structure found inside a larger one, the name of the later is given in parenthesis. Columns $2-4$ give the RA, Dec of the "centre" of each stellar complex and its dimension. Columns 5-7 show the number of SNRs and candidates, stellar associations and number of Nebulae and Henize objects found in each complex. Columns 8 and 9 indicate presence of 8.6-GHz and CO line emission respectively. Finally in Col. 10 the complexes are characterised. I indicates starbursts; II starburst candidates; III active complexes candidates and IV active complexes.

\begin{tabular}{|c|c|c|c|c|c|c|c|c|c|}
\hline Stellar grouping & $\begin{array}{c}\text { RA (1950) } \\
\text { h m s }\end{array}$ & $\begin{array}{c}\text { Dec (1950) } \\
\operatorname{deg} \mathrm{m} \mathrm{s}\end{array}$ & $\begin{array}{l}\text { Dimension } \\
(\mathrm{pc})\end{array}$ & No. SNRs & Association ID & No. Nebulae & $\begin{array}{c}8.6 \\
\mathrm{GHz}\end{array}$ & $\begin{array}{l}\mathrm{CO} \\
\text { line }\end{array}$ & Characterisation \\
\hline SC1 & 05:44:39 & $-67: 15: 18$ & 1421.5 & 1 & 173,257 & 46 & - & - & IV \\
\hline $\mathrm{C} 1$ (SC1) & 05:43:30 & $-67: 17: 29$ & 355.3 & 0 & 173,257 & 57 & - & - & IV \\
\hline A1 & $05: 52: 53$ & $-67: 29: 46$ & 205 & 0 & 173,257 & 22 & - & - & IV \\
\hline A2 & 05:43:44 & $-67: 19: 37$ & 191 & 1 & 173,257 & 31 & - & - & IV \\
\hline $\mathrm{SC} 2$ & $05: 31: 22$ & $-66: 54: 42$ & 1526.5 & 7 & & 24 & - & - & IV \\
\hline $\mathrm{C} 2(\mathrm{SC} 2)$ & $05: 32: 45$ & $-67: 05: 11$ & 749 & 1 & 173 & 41 & - & - & IV \\
\hline C3 (C2) & $05: 32: 47$ & $-66: 58: 22$ & 572 & 1 & & 32 & - & - & IV \\
\hline A3 (SC2) & $05: 29: 45$ & $-66: 37: 17$ & 204 & 0 & & 31 & - & - & IV \\
\hline $\mathrm{A} 4(\mathrm{C} 2)$ & $05: 30: 53$ & $-66: 39: 15$ & 245 & 0 & & 26 & - & - & IV \\
\hline A5 (SC2) & $05: 32: 55$ & $-67: 32: 29$ & 164 & 1 & 173,257 & 41 & - & - & III \\
\hline A6 & $05: 25: 58$ & $-66: 12: 18$ & 299 & 1 & & 20 & $\sqrt{ }$ & $\sqrt{ }$ & II \\
\hline A7 & 05:27:01 & $-67: 27: 42$ & 299 & 1 & 173,257 & 10 & $\sqrt{ }$ & - & II \\
\hline $\mathrm{C} 4$ & $05: 13: 28$ & $-67: 20: 57$ & 314 & 0 & 173,257 & 16 & - & - & IV \\
\hline A8 (C4) & $05: 13: 10$ & $-67: 22: 34$ & 246 & 0 & 173,257 & 16 & - & - & IV \\
\hline A9 & 05:10:55 & $-67: 11: 35$ & 192 & 0 & 173,257 & 26 & - & $\sqrt{ }$ & IV \\
\hline A 10 & 04:57:01 & $-66: 28: 27$ & 245 & 1 & & 7 & $\sqrt{ }$ & $\sqrt{ }$ & I \\
\hline $\mathrm{C} 5$ & $05: 20: 58$ & $-68: 10: 16$ & 765 & 2 & & 17 & $\sqrt{ }$ & $\sqrt{ }$ & II \\
\hline C6 (C5) & $05: 22: 13$ & $-67: 58: 26$ & 342.6 & 1 & 173,257 & 36 & $\sqrt{ }$ & $\sqrt{ }$ & I \\
\hline A11 (C6) & $05: 22: 13$ & $-67: 56: 44$ & 205 & 1 & 173,257 & 17 & $\sqrt{ }$ & $\sqrt{ }$ & I \\
\hline $\mathrm{C} 7$ & 05:07:30 & $-68: 44: 27$ & 545 & 1 & & 39 & $\sqrt{ }$ & $\sqrt{ }$ & II \\
\hline A12 (C7) & 05:09:04 & $-68: 49: 37$ & 287 & 1 & & 17 & $\sqrt{ }$ & $\sqrt{ }$ & I \\
\hline A13 (C7) & 05:05:38 & $-68: 39: 12$ & 245 & 0 & & 27 & - & - & IV \\
\hline A14 & 05:04:00 & $-68: 57: 45$ & 230 & 0 & & 11 & - & - & IV \\
\hline $\mathrm{C} 8$ & $04: 55: 33$ & $-69: 32: 52$ & 628 & 1 & & 12 & - & - & IV \\
\hline A15 (C8) & $04: 56: 55$ & $-69: 30: 18$ & 273 & 1 & & 10 & - & - & IV \\
\hline $\mathrm{C} 9$ & 05:09:26 & $-70: 08: 58$ & 328 & 0 & $150,153,151$ & 19 & - & - & IV \\
\hline $\mathrm{A} 16$ (C9) & 05:08:09 & $-70: 05: 05$ & 246 & 0 & 150,151 & 10 & - & - & IV \\
\hline $\mathrm{SC} 3$ & 05:04:44 & $-70: 29: 23$ & 1093.2 & 2 & $150,153,151$ & 23 & - & - & IV \\
\hline C10 (SC3) & 05:05:01 & $-70: 32: 56$ & 478.3 & 1 & $150,153,151$ & 13 & - & - & IV \\
\hline C11(C10) & 05:06:16 & $-70: 40: 18$ & 328 & 0 & $150,153,151$ & 17 & - & - & IV \\
\hline $\mathrm{C} 12$ & 04:58:02 & $-70: 53: 27$ & 574 & 0 & & 2 & - & - & IV \\
\hline A17 & $04: 56: 28$ & $-71: 28: 37$ & 164 & 0 & & 0 & - & - & IV \\
\hline $\mathrm{C} 13$ & 05:30:55 & $-71: 50: 37$ & 574 & 0 & 76 & 6 & - & - & IV \\
\hline $\mathrm{C} 14$ & $05: 39: 55$ & $-71: 11: 22$ & 328 & 0 & 76 & 10 & - & $\sqrt{ }$ & IV \\
\hline $\mathrm{C} 15$ & $05: 35: 42$ & $-71: 12: 22$ & 437 & 0 & 76 & 17 & - & $\sqrt{ }$ & IV \\
\hline A18 & $05: 31: 26$ & $-71: 06: 11$ & 218.64 & 2 & 76 & 21 & $\sqrt{ }$ & $\sqrt{ }$ & I \\
\hline $\mathrm{C} 16$ & 05:47:50 & $-70: 41: 07$ & 615 & 1 very close & 150,151 & 15 & - & $\sqrt{ }$ & IV \\
\hline A19 (C16) & $05: 47: 53$ & $-70: 44: 31$ & 210 & 0 & $150,153,151$ & 14 & - & $\sqrt{ }$ & IV \\
\hline A 20 & $05: 49: 53$ & $-70: 05: 51$ & 164 & 0 & $150,153,151$ & 10 & - & - & IV \\
\hline $\mathrm{C} 17$ & 05:39:42 & $-69: 21: 58$ & 724 & 10 & 172 & 23 & $\sqrt{ }$ & $\sqrt{ }$ & I \\
\hline C18 (C17) & 05:38:17 & $-69: 26: 16$ & 463 & 3 & 172 & 50 & $\sqrt{ }$ & - & I \\
\hline A21 (C17) & $05: 36: 42$ & $-69: 10: 51$ & 272.3 & 5 & 172 & 29 & $\sqrt{ }$ & - & I \\
\hline A22 (C18) & 05:39:34 & $-69: 28: 00$ & 218 & 1 & 172 & 47 & $\sqrt{ }$ & - & I \\
\hline A23 (C18) & $05: 36: 59$ & $-69: 24: 30$ & 245 & 1 & 172 & 32 & $\sqrt{ }$ & - & I \\
\hline A24 (C17) & $05: 40: 52$ & $-69: 38: 12$ & 298 & 2 & 172 & 44 & $\sqrt{ }$ & - & I \\
\hline A 25 & $05: 35: 48$ & $-68: 55: 28$ & 190.61 & 0 & & 29 & $\sqrt{ }$ & $\sqrt{ }$ & I \\
\hline A26 & $05: 35: 18$ & $-69: 43: 05$ & 231.45 & 0 & 172 & 46 & $\sqrt{ }$ & - & I \\
\hline A27 & $05: 36: 33$ & $-70: 10: 26$ & 272.3 & 0 & $150,153,151$ & 32 & - & - & IV \\
\hline C19 & 05:30:00 & $-69: 11: 52$ & 792.57 & 2 & 172 & 10 & - & - & IV \\
\hline C20 (C19) & $05: 29: 42$ & $-69: 08: 07$ & 517.37 & 2 & 172 & 46 & - & - & IV \\
\hline A28 (C20) & $05: 31: 34$ & $-69: 15: 19$ & 163 & 0 & 172 & 63 & - & - & IV \\
\hline A29 (C20) & $05: 28: 27$ & $-69: 04: 25$ & 285 & 2 & 172 & 71 & - & - & IV \\
\hline $\mathrm{C} 21$ & $05: 26: 03$ & $-69: 51: 28$ & 463 & 1 very close & 172 & 36 & - & - & IV \\
\hline $\mathrm{A} 30(\mathrm{C} 21)$ & $05: 27: 03$ & $-69: 50: 03$ & 163 & 0 & 172 & 28 & - & - & IV \\
\hline $\mathrm{C} 22$ & $05: 19: 27$ & $-69: 34: 39$ & 763 & 2 & 177 & 38 & $\sqrt{ }$ & $\sqrt{ }$ & II \\
\hline A31 (C22) & 05:17:09 & $-69: 31: 39$ & 300 & 0 & 772 & 20 & - & - & III \\
\hline
\end{tabular}


Table 3. List of the derived IRAS fluxes (MJy/sr per pixel), luminocities (in units of solar luminocities) of the identified stellar groupings at 12 , 25, 60 and $100 \mu \mathrm{m}$, the IRAS ratio $F_{60} / F_{100}$ and the flux at $60 \mu \mathrm{m}$ in Jy per pixel.

\begin{tabular}{|c|c|c|c|c|c|c|c|c|c|c|}
\hline Stellar grouping & $f 12$ & $\overline{L L 12}$ & $f 25$ & $\angle 25$ & $f 60$ & 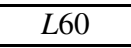 & $\overline{f f 100}$ & $\overline{L L 100}$ & $F_{60} / F_{100}$ & $\bar{F} F_{60}$ \\
\hline SC1 & 0.18 & $2.8 \mathrm{E}+06$ & 0.50 & $3.7 \mathrm{E}+06$ & 3.63 & $1.1 \mathrm{E}+07$ & 8.87 & $1.7 \mathrm{E}+07$ & 0.41 & $0.69 \pm 0.25$ \\
\hline C1 (SC1) & 0.31 & $0.3 E+06$ & 1.02 & $0.5 \mathrm{E}+06$ & 5.38 & $1.1 \mathrm{E}+06$ & 13.38 & $1.6 \mathrm{E}+06$ & 0.40 & $1.02 \pm 0.23$ \\
\hline A1 & 0.36 & $0.1 \mathrm{E}+06$ & 1.28 & $0.2 \mathrm{E}+06$ & 0.67 & $0.4 \mathrm{E}+06$ & 2.16 & $0.8 \mathrm{E}+06$ & 0.31 & $0.13 \pm 0.49$ \\
\hline $\mathrm{A} 2$ & 0.47 & $0.1 \mathrm{E}+06$ & 1.54 & $0.2 \mathrm{E}+06$ & 6.32 & $0.4 \mathrm{E}+06$ & 19.39 & $0.7 \mathrm{E}+06$ & 0.33 & $1.20 \pm 0.23$ \\
\hline $\mathrm{SC} 2$ & 1.14 & $2.0 \mathrm{E}+07$ & 4.46 & $3.8 \mathrm{E}+07$ & 13.96 & $5.0 \mathrm{E}+07$ & 33.50 & $7.2 \mathrm{E}+07$ & 0.42 & $2.65 \pm 0.21$ \\
\hline C2 (SC2) & 0.16 & $0.7 \mathrm{E}+06$ & 0.56 & $1.1 \mathrm{E}+06$ & 4.03 & $3.5 \mathrm{E}+06$ & 9.34 & $4.9 \mathrm{E}+06$ & 0.43 & $0.77 \pm 0.24$ \\
\hline $\mathrm{C} 3(\mathrm{C} 2)$ & 0.11 & $0.3 \mathrm{E}+06$ & 0.52 & $0.6 \mathrm{E}+06$ & 2.08 & $1.1 \mathrm{E}+06$ & 5.13 & $1.5 \mathrm{E}+06$ & 0.41 & $0.40 \pm 0.29$ \\
\hline A3 (SC2) & 0.17 & $0.5 \mathrm{E}+06$ & 1.10 & $0.2 \mathrm{E}+06$ & 0.98 & $0.6 \mathrm{E}+05$ & 3.51 & $0.1 \mathrm{E}+06$ & 0.28 & $0.19 \pm 0.40$ \\
\hline A4 (C2) & 0.12 & $0.6 \mathrm{E}+05$ & 0.89 & $0.2 \mathrm{E}+06$ & 1.06 & $0.1 \mathrm{E}+06$ & 3.46 & $0.2 \mathrm{E}+06$ & 0.31 & $0.20 \pm 0.38$ \\
\hline A5 (SC2) & 1.39 & $0.3 \mathrm{E}+06$ & 3.80 & $0.4 \mathrm{E}+06$ & 30.79 & $1.3 \mathrm{E}+06$ & 64.60 & $0.6 \mathrm{E}+06$ & 0.48 & $5.85 \pm 0.20$ \\
\hline A6 & 1.16 & $0.8 \mathrm{E}+06$ & 2.80 & $0.9 \mathrm{E}+06$ & 25.17 & $3.4 \mathrm{E}+06$ & 58.76 & $4.8 \mathrm{E}+06$ & 0.43 & $4.78 \pm 0.20$ \\
\hline A7 & 0.82 & $0.6 \mathrm{E}+06$ & 2.89 & $0.9 \mathrm{E}+06$ & 27.08 & $3.7 \mathrm{E}+06$ & 50.10 & $4.1 \mathrm{E}+06$ & 0.54 & $5.15 \pm 0.20$ \\
\hline $\mathrm{C} 4$ & 0.68 & $0.5 \mathrm{E}+06$ & 1.53 & $0.6 \mathrm{E}+06$ & 11.15 & $1.7 \mathrm{E}+06$ & 26.38 & $0.4 \mathrm{E}+06$ & 0.42 & $2.12 \pm 0.21$ \\
\hline A8 (C4) & 0.80 & $0.4 \mathrm{E}+06$ & 1.90 & $0.4 \mathrm{E}+06$ & 12.30 & $1.1 \mathrm{E}+06$ & 28.14 & $1.5 \mathrm{E}+06$ & 0.44 & $2.34 \pm 0.21$ \\
\hline A9 & 0.52 & $0.1 \mathrm{E}+06$ & 1.66 & $0.2 \mathrm{E}+06$ & 8.07 & $0.5 \mathrm{E}+06$ & 23.91 & $0.8 \mathrm{E}+06$ & 0.34 & $1.53 \pm 0.22$ \\
\hline A10 & 2.26 & $1.0 \mathrm{E}+06$ & 8.83 & $1.9 \mathrm{E}+06$ & 69.77 & $6.5 \mathrm{E}+06$ & 127.91 & $7.1 \mathrm{E}+06$ & 0.55 & $13.26 \pm 0.20$ \\
\hline C5 & 1.61 & $7.2 \mathrm{E}+06$ & 4.71 & $1.0 \mathrm{E}+07$ & 28.23 & $2.5 \mathrm{E}+07$ & 61.20 & $3.3 \mathrm{E}+07$ & 0.46 & $5.36 \pm 0.20$ \\
\hline C6 (C5) & 0.10 & $0.9 \mathrm{E}+05$ & 6.66 & $2.9 \mathrm{E}+06$ & 54.65 & $9.9 \mathrm{E}+06$ & 97.19 & $1.1 \mathrm{E}+07$ & 0.56 & $10.38 \pm 0.20$ \\
\hline A11 (C6) & 3.21 & $1.0 \mathrm{E}+06$ & 11.89 & $1.8 \mathrm{E}+06$ & 89.85 & $5.8 \mathrm{E}+06$ & 142.78 & $5.6 \mathrm{E}+06$ & 0.63 & $17.07 \pm 0.20$ \\
\hline $\mathrm{C} 7$ & 0.96 & $2.2 \mathrm{E}+06$ & 2.31 & $2.5 \mathrm{E}+06$ & 20.22 & $9.3 \mathrm{E}+06$ & 42.25 & $1.2 \mathrm{E}+07$ & 0.49 & $3.84 \pm 0.21$ \\
\hline A12 (C7) & 1.89 & $1.2 \mathrm{E}+06$ & 5.31 & $1.6 \mathrm{E}+06$ & 42.20 & $5.4 \mathrm{E}+06$ & 79.59 & $6.1 \mathrm{E}+06$ & 0.53 & $8.02 \pm 0.20$ \\
\hline A13 (C7) & 0.59 & $0.3 \mathrm{E}+06$ & 1.56 & $0.4 \mathrm{E}+06$ & 9.20 & $0.8 \mathrm{E}+06$ & 22.76 & $1.3 \mathrm{E}+06$ & 0.40 & $1.75 \pm 0.22$ \\
\hline A14 & 0.94 & $0.4 \mathrm{E}+06$ & 1.90 & $0.4 \mathrm{E}+06$ & 13.16 & $1.1 \mathrm{E}+06$ & 35.27 & $1.7 \mathrm{E}+06$ & 0.37 & $2.50 \pm 0.21$ \\
\hline $\mathrm{C} 8$ & 0.90 & $2.7 \mathrm{E}+06$ & 2.44 & $3.6 \mathrm{E}+06$ & 16.74 & $1.0 \mathrm{E}+07$ & 33.96 & $1.2 \mathrm{E}+07$ & 0.49 & $3.18 \pm 0.21$ \\
\hline A15 (C8) & 0.81 & $0.4 \mathrm{E}+06$ & 1.86 & $0.5 \mathrm{E}+06$ & 11.02 & $1.3 \mathrm{E}+06$ & 25.97 & $1.8 \mathrm{E}+06$ & 0.42 & $2.09 \pm 0.21$ \\
\hline C9 & 1.20 & $1.0 \mathrm{E}+06$ & 3.67 & $1.5 \mathrm{E}+06$ & 9.98 & $1.7 \mathrm{E}+06$ & 25.75 & $2.6 \mathrm{E}+06$ & 0.39 & $1.90 \pm 0.22$ \\
\hline $\mathrm{A} 16$ (C9) & 0.72 & $0.3 \mathrm{E}+06$ & 1.77 & $0.4 \mathrm{E}+06$ & 8.95 & $0.8 \mathrm{E}+06$ & 22.08 & $1.2 \mathrm{E}+06$ & 0.41 & $1.70 \pm 0.22$ \\
\hline SC3 & 0.40 & $3.7 \mathrm{E}+06$ & 0.87 & $3.9 \mathrm{E}+06$ & 3.87 & $7.1 \mathrm{E}+06$ & 9.74 & $1.1 \mathrm{E}+07$ & 0.40 & $0.74 \pm 0.25$ \\
\hline C10 (SC3) & 0.46 & $0.8 \mathrm{E}+06$ & 1.07 & $0.9 \mathrm{E}+06$ & 3.38 & $1.2 \mathrm{E}+06$ & 8.85 & $1.9 \mathrm{E}+06$ & 0.38 & $0.64 \pm 0.25$ \\
\hline C11 (C10) & 0.60 & $0.5 \mathrm{E}+06$ & 1.44 & $0.6 \mathrm{E}+06$ & 4.82 & $0.8 \mathrm{E}+06$ & 12.47 & $1.2 \mathrm{E}+06$ & 0.39 & $0.92 \pm 0.24$ \\
\hline $\mathrm{C} 12$ & 0.37 & $0.9 \mathrm{E}+06$ & 0.87 & $1.1 \mathrm{E}+06$ & 1.82 & $0.9 \mathrm{E}+06$ & 5.75 & $1.8 \mathrm{E}+06$ & 0.32 & $0.35 \pm 0.30$ \\
\hline A17 & 0.69 & $0.1 \mathrm{E}+06$ & 2.14 & $0.2 \mathrm{E}+06$ & 1.89 & $0.8 \mathrm{E}+05$ & 7.51 & $0.2 \mathrm{E}+06$ & 0.25 & $0.36 \pm 0.30$ \\
\hline $\mathrm{C} 13$ & 0.45 & $1.1 \mathrm{E}+06$ & 0.79 & $9.6 \mathrm{E}+05$ & 2.51 & $1.3 \mathrm{E}+06$ & 8.61 & $2.6 \mathrm{E}+06$ & 0.29 & $0.48 \pm 0.27$ \\
\hline $\mathrm{C} 14$ & 0.99 & $0.8 \mathrm{E}+06$ & 1.81 & $0.7 \mathrm{E}+06$ & 10.17 & $1.7 \mathrm{E}+06$ & 31.07 & $3.1 \mathrm{E}+06$ & 0.33 & $1.93 \pm 0.22$ \\
\hline $\mathrm{C} 15$ & 0.62 & $0.9 \mathrm{E}+06$ & 1.13 & $0.8 \mathrm{E}+06$ & 6.19 & $1.8 \mathrm{E}+06$ & 18.46 & $3.3 \mathrm{E}+06$ & 0.34 & $1.18 \pm 0.23$ \\
\hline A18 & 1.86 & $0.7 \mathrm{E}+06$ & 4.59 & $0.8 \mathrm{E}+06$ & 38.36 & $2.8 \mathrm{E}+06$ & 78.27 & $3.5 \mathrm{E}+06$ & 0.49 & $7.29 \pm 0.20$ \\
\hline $\mathrm{C} 16$ & 0.67 & $1.9 \mathrm{E}+06$ & 0.87 & $1.2 \mathrm{E}+06$ & 4.16 & $2.4 \mathrm{E}+06$ & 17.37 & $6.1 \mathrm{E}+06$ & 0.24 & $0.79 \pm 0.24$ \\
\hline A19 (C16) & 1.08 & $0.4 \mathrm{E}+06$ & 1.86 & $0.3 \mathrm{E}+06$ & 4.24 & $0.3 \mathrm{E}+06$ & 21.73 & $0.9 \mathrm{E}+06$ & 0.20 & $0.81 \pm 0.24$ \\
\hline A 20 & 0.05 & $0.1 \mathrm{E}+05$ & 0.01 & $0.1 \mathrm{E}+04$ & 13.07 & $0.5 \mathrm{E}+06$ & 27.62 & $0.7 \mathrm{E}+06$ & 0.47 & $2.48 \pm 0.21$ \\
\hline $\mathrm{C} 17$ & 7.21 & $2.9 \mathrm{E}+07$ & 36.90 & $7.2 \mathrm{E}+07$ & 248.00 & $2.0 \mathrm{E}+08$ & 327.45 & $1.6 \mathrm{E}+08$ & 0.76 & $47.12 \pm 0.20$ \\
\hline C18 (C17) & 3.88 & $6.4 \mathrm{E}+06$ & 16.18 & $1.3 \mathrm{E}+07$ & 149.37 & $4.9 \mathrm{E}+07$ & 222.33 & $4.4 \mathrm{E}+07$ & 0.67 & $28.38 \pm 0.20$ \\
\hline A21 (C7) & 7.62 & $4.3 \mathrm{E}+06$ & 43.93 & $1.2 \mathrm{E}+07$ & 293.57 & $3.4 \mathrm{E}+07$ & 369.90 & $2.5 \mathrm{E}+07$ & 0.79 & $55.78 \pm 0.20$ \\
\hline A22 (C18) & 4.26 & $1.6 \mathrm{E}+06$ & 15.66 & $2.8 \mathrm{E}+06$ & 153.80 & $1.1 \mathrm{E}+07$ & 238.40 & $1.0 \mathrm{E}+07$ & 0.65 & $29.22 \pm 0.20$ \\
\hline A23 (C18) & 2.30 & $1.1 \mathrm{E}+06$ & 8.35 & $1.9 \mathrm{E}+06$ & 77.75 & $7.2 \mathrm{E}+06$ & 128.05 & $7.1 \mathrm{E}+06$ & 0.61 & $14.77 \pm 0.20$ \\
\hline A24 & 5.50 & $3.8 \mathrm{E}+06$ & 21.67 & $7.1 \mathrm{E}+06$ & 190.57 & $2.6 \mathrm{E}+07$ & 276.81 & $2.3 \mathrm{E}+07$ & 0.69 & $36.20 \pm 0.20$ \\
\hline A 25 & 1.42 & $0.4 \mathrm{E}+06$ & 4.05 & $0.5 \mathrm{E}+06$ & 43.42 & $2.4 \mathrm{E}+06$ & 75.47 & $2.5 \mathrm{E}+06$ & 0.57 & $8.25 \pm 0.20$ \\
\hline A26 & 2.62 & $1.1 \mathrm{E}+06$ & 7.49 & $1.5 \mathrm{E}+06$ & 73.60 & $6.1 \mathrm{E}+06$ & 126.18 & $6.3 \mathrm{E}+06$ & 0.58 & $13.98 \pm 0.20$ \\
\hline $\mathrm{A} 27$ & 1.64 & $0.9 \mathrm{E}+06$ & 2.51 & $0.7 \mathrm{E}+06$ & 24.35 & $2.8 \mathrm{E}+06$ & 63.05 & $4.3 \mathrm{E}+06$ & 0.39 & $4.63 \pm 0.20$ \\
\hline C19 & 0.77 & $3.7 \mathrm{E}+06$ & 1.87 & $4.3 \mathrm{E}+06$ & 16.38 & $1.6 \mathrm{E}+07$ & 32.32 & $1.9 \mathrm{E}+07$ & 0.51 & $3.11 \pm 0.21$ \\
\hline C20 (C19) & 0.79 & $1.6 \mathrm{E}+06$ & 1.90 & $1.9 \mathrm{E}+07$ & 14.00 & $5.8 \mathrm{E}+06$ & 28.65 & $7.1 \mathrm{E}+06$ & 0.49 & $2.66 \pm 0.21$ \\
\hline A28 (C20) & 1.30 & $0.3 \mathrm{E}+06$ & 3.42 & $0.3 \mathrm{E}+06$ & 19.26 & $0.8 \mathrm{E}+06$ & 39.05 & $1.0 \mathrm{E}+06$ & 0.49 & $3.66 \pm 0.21$ \\
\hline A29 (C20) & 1.01 & $0.6 \mathrm{E}+06$ & 2.66 & $0.8 \mathrm{E}+06$ & 14.18 & $1.8 \mathrm{E}+06$ & 29.49 & $2.2 \mathrm{E}+06$ & 0.48 & $2.69 \pm 0.21$ \\
\hline $\mathrm{C} 21$ & 1.02 & $1.7 \mathrm{E}+06$ & 1.74 & $1.4 \mathrm{E}+06$ & 14.49 & $4.8 \mathrm{E}+06$ & 32.92 & $6.6 \mathrm{E}+06$ & 0.44 & $2.75 \pm 0.21$ \\
\hline A30 (C21) & 1.01 & $0.2 \mathrm{E}+06$ & 2.40 & $0.2 \mathrm{E}+06$ & 7.42 & $0.3 \mathrm{E}+06$ & 22.65 & $5.6 \mathrm{E}+06$ & 0.33 & $1.41 \pm 0.22$ \\
\hline $\mathrm{C} 22$ & 1.28 & $5.7 \mathrm{E}+06$ & 2.41 & $5.2 \mathrm{E}+06$ & 26.67 & $2.4 \mathrm{E}+07$ & 50.59 & $2.7 \mathrm{E}+07$ & 0.53 & $5.07 \pm 0.20$ \\
\hline A31 (C22) & 1.53 & $1.0 \mathrm{E}+06$ & 3.05 & $1.0 \mathrm{E}+06$ & 31.41 & $4.4 \mathrm{E}+06$ & 56.30 & $4.7 \mathrm{E}+06$ & 0.56 & $5.98 \pm 0.20$ \\
\hline
\end{tabular}

